Marquette University

e-Publications@Marquette

$7-2009$

\title{
Fire retardancy of bis[2-(methacryloyloxy)ethyl] phosphate modified poly(methyl methacrylate) nanocomposites containing layered double hydroxide and montmorillonite
}

\author{
Linjiang Wang \\ Guilin University of Technology \\ Shengpei Shu \\ Hunan Normal University \\ Dan Chen \\ Hunan Normal University \\ Charles A. Wilkie \\ Marquette University, charles.wilkie@marquette.edu
}

Follow this and additional works at: https://epublications.marquette.edu/chem_fac

Part of the Chemistry Commons

\section{Recommended Citation}

Wang, Linjiang; Shu, Shengpei; Chen, Dan; and Wilkie, Charles A., "Fire retardancy of bis[2-(methacryloyloxy)ethyl] phosphate modified poly(methyl methacrylate) nanocomposites containing layered double hydroxide and montmorillonite" (2009). Chemistry Faculty Research and Publications. 88. https://epublications.marquette.edu/chem_fac/88 
Marquette University

\title{
e-Publications@Marquette
}

\section{Chemistry Faculty Research and Publications/College of Arts and Science}

This paper is NOT THE PUBLISHED VERSION; but the author's final, peer-reviewed manuscript.

The published version may be accessed by following the link in the citation below.

Polymer Degradation and Stability, Vol. 94, No. 7 (July, 2009): 1110-1118. DOI. This article is (C) Elsevier and permission has been granted for this version to appear in $\underline{\mathrm{e}-}$

Publications@Marquette. Elsevier does not grant permission for this article to be further copied/distributed or hosted elsewhere without the express permission from Elsevier.

Fire retardancy of bis[2-

(methacryloyloxy)ethyl] phosphate

modified poly(methyl methacrylate)

nanocomposites containing layered double

hydroxide and montmorillonite

\author{
Linjiang Wang \\ Department of Chemistry and Fire Retardant Research Facility, Marquette University, \\ Milwaukee WI \\ Department of Material and Chemistry Engineering, Guilin University of Technology, China \\ Shengpei Su \\ Department of Chemistry and Chemical Engineering, Hunan Normal University, China
}




\title{
Dan Chen
}

Department of Chemistry and Chemical Engineering, Hunan Normal University, China Charles A. Wilkie

Department of Chemistry and Fire Retardant Research Facility, Marquette University, Milwaukee WI

\begin{abstract}
Copolymer nanocomposites were prepared by suspension copolymerization of bis[2-

(methacryloyloxy)ethyl] phosphate and methyl methacrylate, together with bis(2-ethylhexyl) phosphate layered double hydroxide and a montmorillonite, Cloisite 93A. X-ray diffraction and transmission electron microscopy were used to characterize the morphology of nanocomposites and the dispersion of additives in the polymer. The thermal stability of the nanocomposites has been assessed by thermogravimetric analysis and cone calorimetry has been used to study the fire properties. Bis[2-(methacryloyloxy)ethyl] phosphate not only copolymerized with MMA, but also aids in the dispersion of additives in PMMA. The copolymer nanocomposites have better dispersion and higher degradation temperature and more char mass than the corresponding PMMA nanocomposites. The largest peak reduction in the heat release rate of the copolymer nanocomposites are 52 and $65 \%$ for LDH and MMT additives, respectively.
\end{abstract}

\section{Keywords}

Nanocomposites; Layered double hydroxides; PMMA; Fire retardancy

\section{Introduction}

Poly(methyl methacrylate) (PMMA), a hard, rigid, transparent, and rather fragile polymer, is widely used, mainly due to its good optical properties, outstanding mechanical properties, high thermal stability, and excellent electric performance. On the other hand, it is known that PMMA is highly combustible and flame retardation is needed for many applications.

Phosphorus-containing compounds are effective flame-retardantadditives. The use of phosphorus compounds as flame-retardant additives in polymers is well-established ${ }^{[1]}$, [2]. However, the addition of additives has disadvantages, since additives often have to be used in relatively high concentrations, leading to concomitant undesirable changes in physical and mechanical properties; additives may be leached or lost from the polymer during service, posing a potential environmental hazard ${ }^{[3]}$.

The use of reactive flame retardants, i.e., phosphorus-containing flame-retardant groups that are inherently part of the polymer backbone or covalently attached as side groups to the polymer chain, may promote cross-linking and char formation during combustion; even low concentrations may suffice to provide adequate protection, and reactive flame retardants are less likely to be lost from the polymer during service.

Phosphorus-containing components have been used in the synthesis of several flame-retardant for polyesters ${ }^{[4],[5]}$, polyurethanes ${ }^{[6],[7]}$, and epoxy resins ${ }^{[8],[9]}$. Price and coworkers synthesized several phosphorus-containing monomers that could be combined with either methyl 
methacrylate or styrene to obtain phosphorus-containing polymers which had enhanced fire retardancy compared to the pristine polymers ${ }^{[10],},[11]$.

During the past decade, a significant amount of work has been devoted to the utilization of organicallymodified clays to prepare polymer-clay nanocomposites which can have a very significant effect on many properties, including fire, mechanical and barrier properties of the polymer. A recent book reviews the use of nano-dimensional materials in polymers ${ }^{[12]}$. As fire retardants, these materials bring about a reduction in the heat release rate which has been ascribed to the formation of a barrier to mass transport and to insulate the polymer from the heat source $\frac{[13],[14]}{}$.

Among the nano-dimensional materials, most attention has been directed toward montmorillonite (MMT) [14], [15], [16], [17], since it can enhance the mechanical and fire properties, and it is commercially available and well-studied. Layered double hydroxides (LDHs) are another interesting material for nanocomposite formation. These have received considerable attention in recent years because of their special structure and properties, ease of synthesis in the laboratory, and their potential industrial uses, including as fire retardants $[18],[19]$. The utility of $\underline{\underline{L D H s}}$ comes about since one can vary the identity of the metals, the anions and the stoichiometry to ascertain how these affect the ability of the nano-material to disperse in a polymer and to determine what effect dispersion has on the fire properties of the polymer [20], [21], [22], [23], [24].

Previous work from these laboratories showed that when $5 \%$ of a phosphate-containing LDH was used, one could obtain a $33 \%$ reduction in the peak heat release rate, $\mathrm{PHRR}^{[23]}$, while Price et al. obtained a $30 \%$ reduction for the phosphorus-containing methacrylate copolymer alone ${ }^{[10], ~[11]}$. The raison d'être for this work is to combine these two materials to investigate possible synergistic interactions between the additive and the copolymer.

\section{Experimental}

\subsection{Materials}

$\mathrm{Al}\left(\mathrm{NO}_{3}\right)_{3} \cdot 9 \mathrm{H}_{2} \mathrm{O}$ (reagent grade), $\mathrm{Mg}\left(\mathrm{NO}_{3}\right)_{2} \cdot 6 \mathrm{H}_{2} \mathrm{O}$ (reagent grade), $\mathrm{NaOH}$ (reagent grade), poly(methyl methacrylate) (reagent grade), with typical $M_{\mathrm{w}} 120,000$, monomeric methyl methacrylate, initiator benzoyl peroxide (BPO) (97\%), bis[2-(methacryloyloxy)ethyl] phosphate were purchased from the Aldrich Chemical Company. Sodium bis(2-ethylhexyl) phosphate (reagent grade 97\%) was obtained from TCl America. Polyvinyl alcohol (PVA) $\left(\mathrm{M}_{\mathrm{w}}=22,000\right)$ was acquired from

Acros. Montmorillonite (MMT, Cloisite 93A), with basal spacing $2.24 \mathrm{~nm}$, was obtained from Southern Clay Products, Inc.

\subsection{Synthesis of phosphate-LDH}

The phosphate-LDH was prepared by the co-precipitationmethod of Zhao et al. with modification $\frac{[25]}{}$. The process has been previously described $\underline{[23]}$.

\subsection{Synthesis of copolymer-clay nanocomposites}

The copolymers of MMA and copolymer-clay nonocomposites were synthesized following a literature suspension polymerization method with minor modifications $\frac{[15]}{}$. In a $3000 \mathrm{~mL}$ round bottom flask were placed $1000 \mathrm{~mL} \mathrm{H}{ }_{2} \mathrm{O}$, and $1 \mathrm{~g}$ poly(vinyl alcohol) $\mathrm{PVOH}$, and this mixture was stirred for $1 \mathrm{~h}$ at $95^{\circ} \mathrm{C}$, then the temperature was decreased to $85^{\circ} \mathrm{C}$. In a $500 \mathrm{~mL}$ beaker were placed $200 \mathrm{~mL}$ 
MMA monomer, $1 \mathrm{~g} \mathrm{BPO}$, and $52 \mathrm{~mL}$ bis[2-(methacryloyloxy)ethyl]phosphate. After this mixture was cooled to $0{ }^{\circ} \mathrm{C}, 6 \mathrm{~g}, 10 \mathrm{~g}$, or $20 \mathrm{~g}$ of phosphate-LDH or MMT Cloisite 93A was added with magnetic stirring to obtain a homogeneously dispersed system. The clay-MMA-initiator mixture was added dropwise to the water-PVOH system, and the contents were stirred at $75{ }^{\circ} \mathrm{C}$ for $2 \mathrm{~h}, 85^{\circ} \mathrm{C}$ for $2 \mathrm{~h}$ and then $95^{\circ} \mathrm{C}$ for $2 \mathrm{~h}$. The system was allowed to cool for $1 \mathrm{~h}$ with stirring and sit for $24 \mathrm{~h}$. The composites were repeatedly washed with water and methanol, air-dried and then dried in a vacuum oven at $100^{\circ} \mathrm{C}$ for $24 \mathrm{~h}$. The reaction products are identified as SusCoPMMA, SusCoPMMA + LDH and SusCoPMMA + MMT. The PMMA homopolymer and its nanocomposites were synthesized by a similar method without the addition of the phosphate-containing comonomer. These reaction products are identified as SusPMMA, SusPMMA + LDH and SusPMMA + MMT. The phosphorus content of suspension copolymerized PMMA and its char after calcination at $800^{\circ} \mathrm{C}$ for $12 \mathrm{~h}$ are $5.9 \%$ and $8.1 \%$, respectively.

\subsection{Characterization}

The phosphorus content in the polymers and the chars after combustion, was determined by the molybdovanadophosphonic acid method 26$]$. X-ray diffraction (XRD) was performed on a Rigaku Miniflex II desktop X-ray powder diffractometer with CuK $\alpha$ generator $(\lambda=0.15404 \mathrm{~nm})$; the accelerating voltage was $50 \mathrm{kV}$ at a current of $20 \mathrm{~mA}$. Scans were taken at $2 \vartheta=2-70^{\circ}$ at 0.1 step size. Polymer composite samples were pressed into $20 \times 15 \times 1 \mathrm{~mm}^{3}$ thick plaques by compression molding. The transmission electron microscopy (TEM) images were carried out using a JEOL 1230 transmission electron microscope at an accelerating voltage of $100 \mathrm{kV}$. Thermogravimetric analysis (TGA) was performed on an SDT 2960 instrument at the 12-14 mg scale under a flowing nitrogen atmosphere at a ramp rate of $20^{\circ} \mathrm{C} / \mathrm{min}$. All TGA samples were run in duplicate and the average values are reported; the reproducibility of temperature is $\pm 2{ }^{\circ} \mathrm{C}$ and mass is reproducible to $\pm 0.4 \%$. Cone calorimetry was performed on an Atlas CONE-2 according to ASTM E 1354 at an incident flux of $50 \mathrm{~kW} / \mathrm{m}^{2}$ using a cone shaped heater, exhaust flow was set at $24 \mathrm{~L} / \mathrm{s}$; all samples were burned in triplicate. Cone samples (about $30 \mathrm{~g}$ ) were prepared by compression molding into $100 \times 100 \times 3 \mathrm{~mm}^{3}$ square plaques. Based on thousands of samples that have been run, cone measurements are considered to have error bars of $\pm 10 \%$.

\section{Results and discussion}

\subsection{Morphological characterization}

The morphology of these materials was evaluated by the combination of X-ray diffraction (XRD) and transmission electron microscopy (TEM). From XRD measurements, one can either observe a shift and/or broadening, or the disappearance of the peak. If a peak is observed shifted to lower values of $2 \vartheta$, this is typically indicative of intercalation. If the peak does not shift and remains sharp, this likely means that the clay is simply present as an additive without any enlargement of the gallery space. Finally, if the peak vanishes or is significantly broadened, this is indicative of some disordering which could either suggest disordering without insertion of the polymer between the clay layers, a microcomposite, or disordering accompanied by polymer insertion, exfoliation.

The X-ray traces of the PMMA nanocomposite synthesized with the different additives are shown in Fig. 1. Two types of additives, phosphate LDH (Fig. 1A and B) and montmorillonite Cloisite 93A (Fig. 1C and $D)$, were used in the preparation of nanocomposites. From Fig. $1 \mathrm{~A}$, the basal spacing $\left(d_{003}\right)$ of HDEHP-LDH, $2.34 \mathrm{~nm}$, does not change upon suspension polymerization of the 
PMMA homopolymer nanocomposite, but the peak is weak, indicative of disordering. The same result is seen for the copolymer, Fig. 1B; the XRD results indicate that TEM is required to evaluate the morphology of these systems.
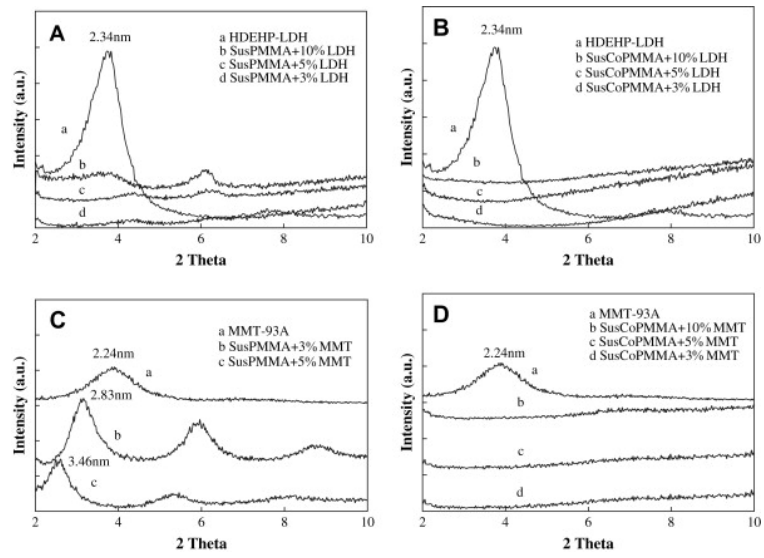

Fig. 1. XRD traces of suspension polymerized MMA with HDEHP-LDH (A); suspension copolymerization of MMA with the phosphorus-containing comonomer (B); suspension polymerization of MMA with MMT (C); and (D) suspension copolymerization of MMA with the phosphorus-containing comonomer and MMT (D).

For the montmorillonite series (Fig. 1C and D), the first order basal reflection of montmorillonite Cloisite $93 \mathrm{~A}$ corresponds to a basal spacing of $2.24 \mathrm{~nm}$. Upon suspension polymerization at $3 \%$ additive loading, the (001) basal reflection is sharper and shifts to $2.83 \mathrm{~nm}$. At 5\% Cloisite 93A loading, the (001) basal reflection is again broader than in the pristine clay, but not as sharp as at $3 \%$ loading and the peak is shifted to $3.46 \mathrm{~nm}$ (Fig. 1C). Second order reflections, indicative of increased ordering, are also seen. The indication from XRD is that intercalated systems have been produced, possibly with some disordering. It was not possible to synthesize a PMMA nanocomposite containing 10\% MMT because this large an amount of MMT cannot be well-dispersed in the PVOH solution of the monomer. When the two comonomers, phosphate-containing and MMA, were solution polymerized in the presence of 3,5 and 10\% Cloisite 93A loading, the (001) basal reflection disappeared entirely (Fig. 1D), which again indicates the need for TEM. It is interesting to note that when the phosphorus-containing comonomer is present, one can disperse $10 \%$ clay.

Typically two TEM images are presented, one at low magnification to see the global dispersion of the clay in the polymer and one at high magnification to permit a description of the system. Fig. 2 shows the TEM images of phosphate-containing LDHs in PMMA (Fig. 2 top) and in the copolymer (Fig. 2 bottom). From the lower magnification images, on the left, the global dispersion is better in the copolymer than in PMMA, but both of these should be described as microcomposites. 


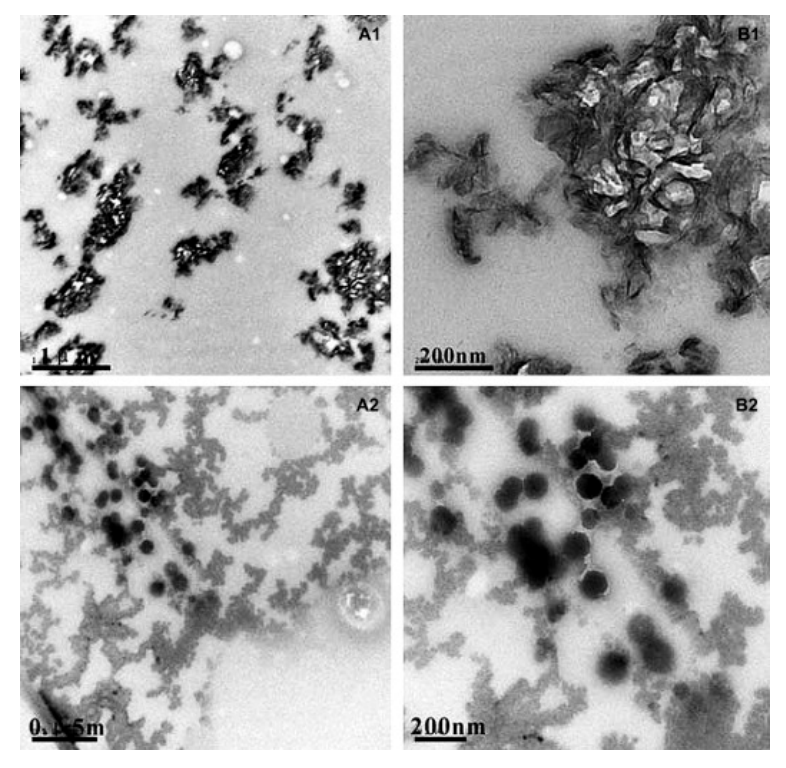

Fig. 2. TEM images for suspension polymerization of PMMA with HDEHP-LDH; $(A 1, B 1)$ and suspension polymerization of MMA with the phosphate-containing comonomer with HDEHP-LDH (A2, B2).

The TEM images of the MMT systems are shown in Fig. 3. For both PMMA and the copolymer nanocomposites, the low magnification images show that good dispersion has been achieved. In the right top of Fig. 3 , labeled as B3, one can see the intercalated nature of this PMMA-MMT nanocomposite. For the copolymer-MMT system, the high magnification image, labeled B4, shows the random arrangement of the clay layers, indicative of exfoliation. These TEM results are consistent with the $X R D$ results.

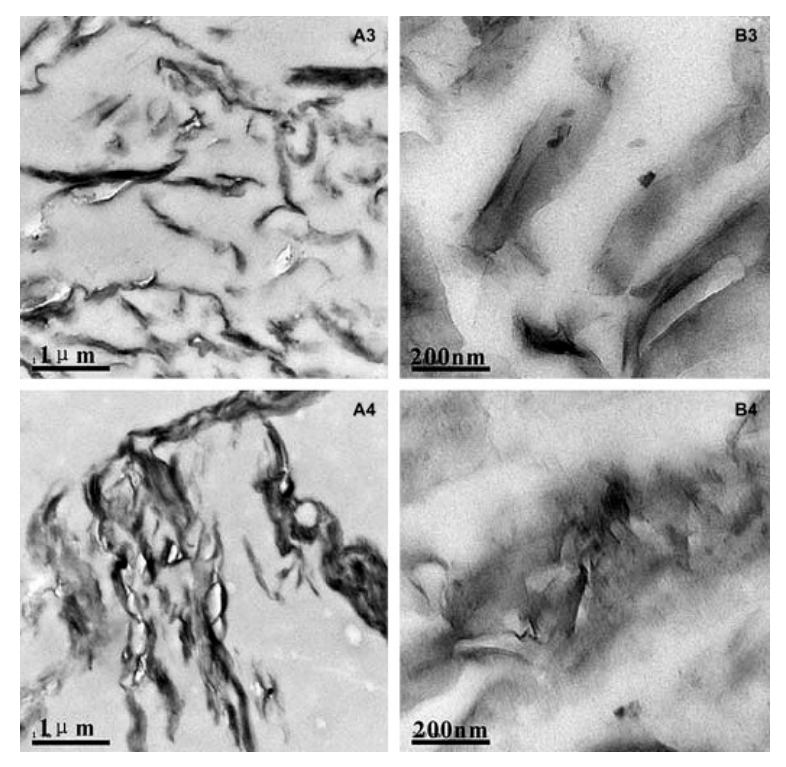

Fig. 3. TEM images of suspension polymerization of MMA with MMT (A3, B3) and MMT and suspension polymerization of MMA with the phosphate-containing comonomer with MMT (A4, B4). 


\subsection{Thermal degradation behavior}

Thermogravimetric analysis (TGA) of polymers enables a description of the degradation process. The points of interest are the onset temperature of the degradation, typically taken as the point at which $10 \%$ degradation occurs, $T_{0.1}$, the mid-point of the degradation, $T_{0.5}$, and the fraction of char which remains at the conclusion of the run. All of this data is reported in Table 1 and the TGA curves as well as the derivatives, DTG, are shown in Fig. 4 , Fig. 5.

Table 1. TGA summary results for methacrylate and methacrylate (nano)composites.

\begin{tabular}{|l|l|l|l|}
\hline Formulation & $T_{0.1}\left({ }^{\circ} \mathrm{C}\right)$ & $T_{0.5}\left({ }^{\circ} \mathrm{C}\right)$ & Char $(\%)$ \\
\hline Pure PMMA & 276 & 368 & 0.6 \\
\hline Sus PMMA & 270 & 367 & 0 \\
\hline Sus PMMA + 5\% LDH & 286 & 326 & 2.1 \\
\hline Sus CoPMMA & 332 & 409 & 2.0 \\
\hline Sus CoPMMA + 5\% LDH & 309 & 403 & 8.2 \\
\hline Sus PMMA + 5\% MMT & 303 & 389 & 7.5 \\
\hline Sus CoPMMA + 5\% MMT & 314 & 411 & 11.5 \\
\hline
\end{tabular}
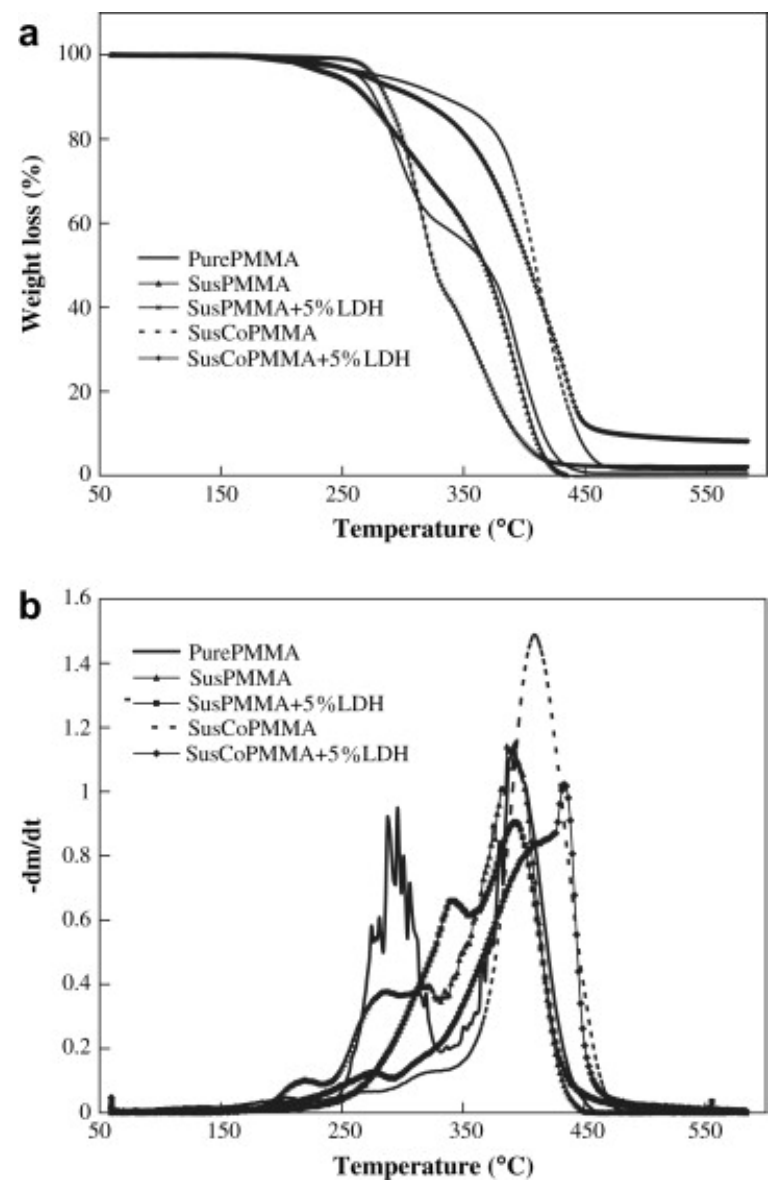

Fig. 4. TGA (a) and DTG (b) curves of PMMA phosphate-LDH nanocomposites. 

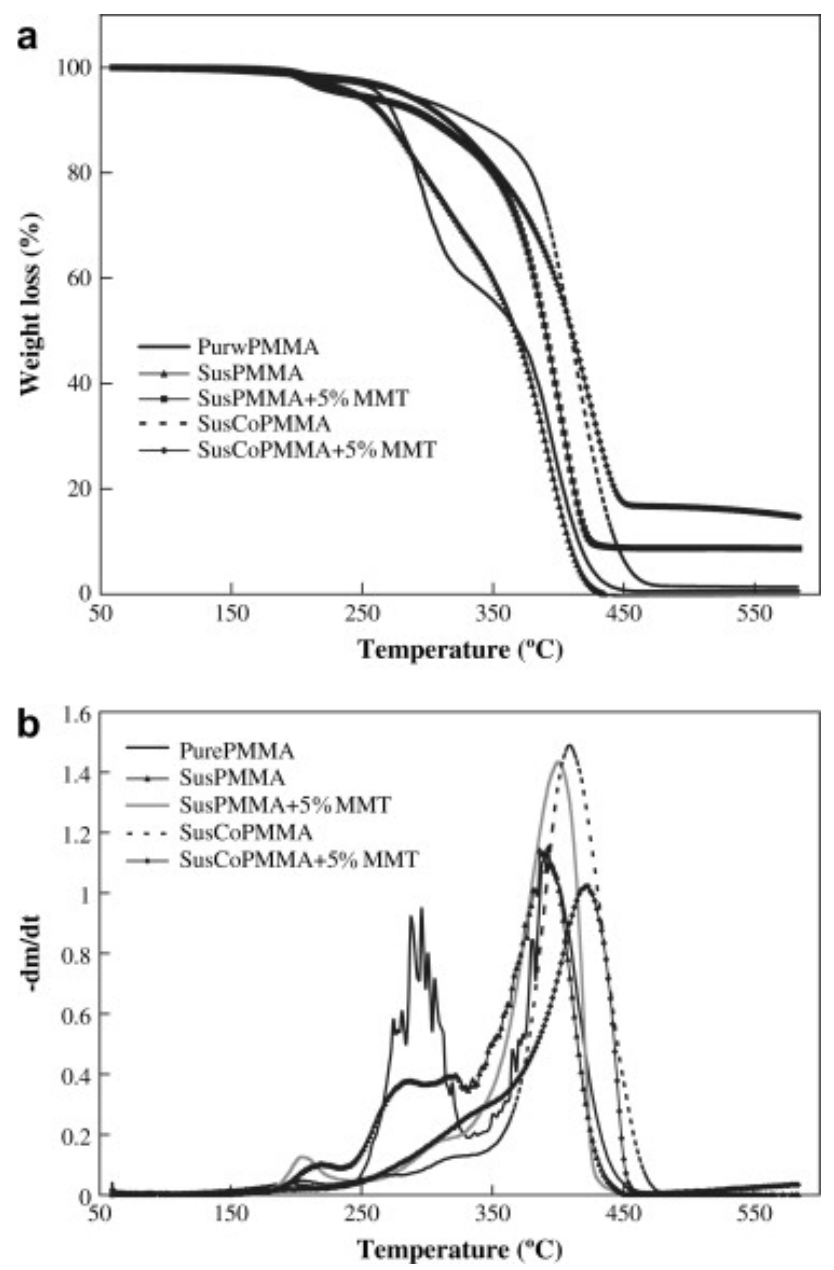

Fig. 5. TGA (a) and DTG (b) curves of PMMA-MMT nanocomposites (B).

The thermal degradation of PMMA is well-understood and consists of three steps, attributed to cleavage of weak links, i.e., head-to-head linkages $\stackrel{[27]}{ }$, and then a larger mass loss due to end chain unsaturation and the final event, which accounts for the majority of the degradation, is due to random scission $\underline{[28]}$.

The copolymer and copolymer nanocomposites begin thermal degradation above $250{ }^{\circ} \mathrm{C}$, and at a much slower rate than the melt blended commercial PMMA. The degradation rapidly increases after $350^{\circ} \mathrm{C}$, which is much higher than that of pristine PMMA. The onset and $50 \%$ degradation temperatures for the copolymer are $60^{\circ}$ and $40^{\circ}$ higher than the corresponding suspension polymerized PMMA, indicating substantial enhanced thermal stability for the copolymer. The addition of either MMT or LDH at 5\% loading to this copolymer brings about a reduction in the onset temperature, probably due to the presence of the less stable additive.

\subsection{Fire retardancy of the nanocomposites}

Cone calorimetry is one of the most useful methods to evaluate the fire performance of polymers. The parameters that may be evaluated from cone calorimetry include the heat release rate (HRR), and especially its peak value (PHRR); the time to ignition ( $\left.t_{\text {ig }}\right)$; the volume of smoke (VOS); the total heat released, (THR), a measure of the extent to which the entire polymer burns; and the average mass loss rate (AMLR). The peak heat release rate, which gives information about the size of the fire and can 
be viewed as the "driving force" of the fire, is considered to be one of the most important parameters in assessing potential behavior in a real fire.

The cone calorimetric data, obtained at heat flux of $50 \mathrm{~kW} / \mathrm{m}^{2}$, are given in Table 2 while the heat release rate plots are shown in Fig. 6 . In addition, Fig. 7 shows the total heat released as a function of time while Fig. 8 provides the smoke data. Both pristine, commercial PMMA and the material which was obtained by suspension polymerization give similar results but the copolymer shows a $44 \%$ reduction in the PHRR, compared to PMMA. As noted in the experimental section, the copolymer contained $5.9 \%$ phosphorus as determined by analysis. For comparison, Price et al. used a copolymer which contained $3.5 \%$ phosphorus, based upon the amounts of starting material, and observed a $30 \%$ reduction in the PHRR at $35 \mathrm{~kW} / \mathrm{m}^{2}$. It is commonly agreed that fire retardancy due to phosphorus is

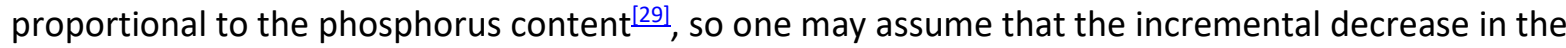
PHRR seen in this study is due to the increased phosphorus content. The total heat released is less for the copolymer compared to the homopolymer, which may be an indication that some of the material does not burn. Strikingly, the volume of smoke is approximately four times larger for the copolymer than for the homopolymer. Once again, this may likely be attributed to the presence of phosphorus.

Table 2. Cone calorimetric data for methacrylate and methacrylate (nano)composites.

\begin{tabular}{|c|c|c|c|c|c|c|c|}
\hline Formulation & $\begin{array}{l}\text { PHRR } \\
\left(\mathrm{kW} / \mathrm{m}^{2}\right)\end{array}$ & Reduction(\%) & $\begin{array}{l}\text { THR } \\
\left(\mathrm{mJ} / \mathrm{m}^{2}\right)\end{array}$ & $\operatorname{VOS}(\mathrm{L})$ & $\begin{array}{l}\text { AMLR } \\
\left(\mathrm{g} / \mathrm{s} \mathrm{m}^{2}\right)\end{array}$ & $t_{\text {ig }}(\mathrm{S})$ & $t_{\text {PHRR }}(\mathrm{S})$ \\
\hline Pure PMMA & $1129 \pm 95$ & NA & $86 \pm 1$ & $361 \pm 25$ & $32 \pm 4$ & $9 \pm 2$ & $72 \pm 7$ \\
\hline Sus PMMA & $1081 \pm 63$ & 4 & $81 \pm 1$ & $376 \pm 2$ & $32 \pm 1$ & $7 \pm 1$ & $76 \pm 14$ \\
\hline Sus CoPMMA & $635 \pm 41$ & 44 & $60 \pm 1$ & $1659 \pm 8$ & $34 \pm 16$ & $8 \pm 1$ & $62 \pm 3$ \\
\hline Sus PMMA p 3\% LDH & $798 \pm 76$ & 29 & $83 \pm 3$ & $595 \pm 100$ & $27 \pm 1$ & $8 \pm 2$ & $\begin{array}{l}101 \pm \\
10\end{array}$ \\
\hline Sus PMMA p 5\% LDH & $623 \pm 42$ & 45 & $73 \pm 3$ & $747 \pm 15$ & $22 \pm 2$ & $6 \pm 1$ & $80 \pm 6$ \\
\hline Sus PMMA p 10\% LDH & $491 \pm 18$ & 56 & $73 \pm 1$ & $797 \pm 60$ & $17 \pm 0$ & $7 \pm 1$ & $65 \pm 15$ \\
\hline Sus CoPMMA p 3\% LDH & $720 \pm 28$ & 36 & $61 \pm 1$ & $1595 \pm 30$ & $28 \pm 1$ & $\begin{array}{l}10 \pm \\
1\end{array}$ & $58 \pm 28$ \\
\hline Sus CoPMMA p 5\% LDH & $680 \pm 24$ & 40 & $57 \pm 1$ & $1727 \pm 80$ & $26 \pm 1$ & $9 \pm 1$ & $61 \pm 1$ \\
\hline $\begin{array}{l}\text { Sus CoPMMA p } 10 \% \\
\text { LDH }\end{array}$ & $542 \pm 20$ & 52 & $63 \pm 2$ & $1647 \pm 50$ & $19 \pm 1$ & $\begin{array}{l}11 \pm \\
0\end{array}$ & $60 \pm 6$ \\
\hline Sus PMMA p 3\% MMT & $539 \pm 58$ & 52 & $69 \pm 1$ & $730 \pm 80$ & $17 \pm 1$ & $5 \pm 2$ & $58 \pm 4$ \\
\hline Sus PMMA p 5\% MMT & $538 \pm 28$ & 52 & $66 \pm 6$ & $654 \pm 50$ & $17 \pm 0$ & $6 \pm 2$ & $56 \pm 9$ \\
\hline $\begin{array}{l}\text { Sus CoPMMA p 3\% } \\
\text { MMT }\end{array}$ & $439 \pm 15$ & 61 & $57 \pm 1$ & $\begin{array}{l}1611 \pm \\
110\end{array}$ & $13 \pm 5$ & $\begin{array}{l}11 \pm \\
3\end{array}$ & $87 \pm 5$ \\
\hline $\begin{array}{l}\text { Sus CoPMMA p 5\% } \\
\text { MMT }\end{array}$ & $482 \pm 54$ & 57 & $61 \pm 2$ & $1455 \pm 55$ & $17 \pm 2$ & $\begin{array}{l}14 \pm \\
1\end{array}$ & $81 \pm 12$ \\
\hline $\begin{array}{l}\text { Sus CoPMMA p } 10 \% \\
\text { MMT }\end{array}$ & $392 \pm 131$ & 65 & $51 \pm 17$ & $1412 \pm 30$ & $21 \pm 7$ & $\begin{array}{l}15 \pm \\
3\end{array}$ & $85 \pm 8$ \\
\hline
\end{tabular}



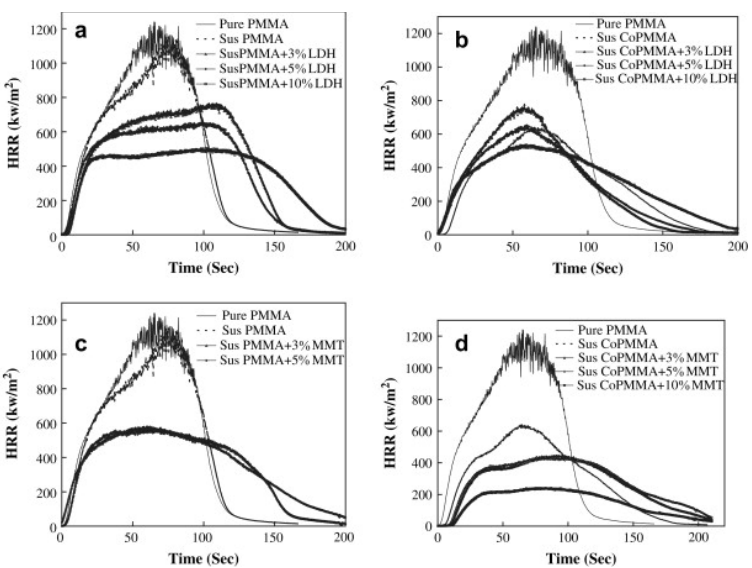

Fig. 6. HRR vs. time for (a) suspension polymerization PMMA LDH nanocomposites, (b) suspension copolymerization PMMA LDH nanocomposites, (c) suspension polymerization PMMA-MMT nanocomposites and (d) suspension copolymerization nanocomposites.

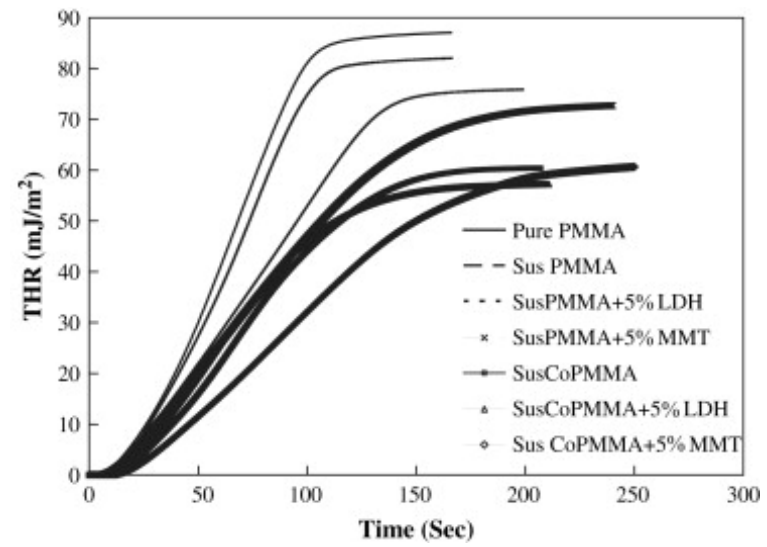

Fig. 7. THR vs. time for suspension polymerization and suspension copolymerization nanocomposites.

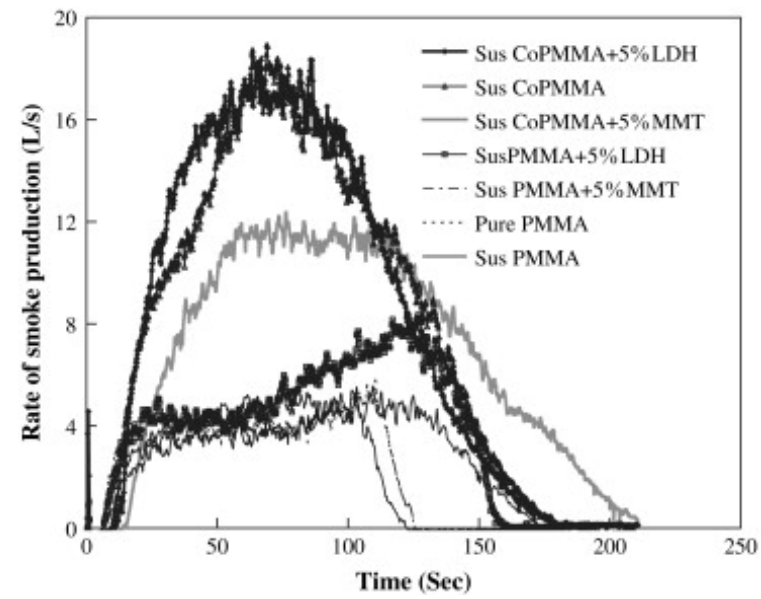

Fig. 8. Smoke evolution vs. time for suspension polymerizationand suspension copolymerization nanocomposites.

In previous work for these laboratories $\stackrel{[23]}{ }$, nanocomposites of PMMA with the phosphate-containing LDH have been prepared both by melt blending and by bulk polymerization. At $10 \% \mathrm{LDH}$, the reduction in the PHRR by melt blending was $37 \%$ vs. $31 \%$ by bulk polymerization and $56 \%$ in this work. By melt 
blending, a mixed intercalated/exfoliated nanocomposite was produced but bulk polymerization gave a microcomposite and, in this work, a microcomposite was also obtained. It is obvious that there is no correlation between dispersion of the polymer in the clay and reduction in PHRR but such a correlation is well-known for MMT systems. Fire retardancy due to the addition of an LDH to a polymer may be ascribed to 1) endothermic decomposition of the constituent hydroxides, 2) the presence of water amongst the combustible gases, and 3) the presence of the oxide char formed during the degradation.

Comparing suspension polymerized PMMA with the copolymer in the presence of the LDH, there is very little difference in the PHRR. While the presence of the phosphorus comonomer has a significant effect for the polymer only, when the LDH is added, there is no additional effect. There is a smooth decrease in both the mass loss rates and the PHRR as the amount of LDH increases, in agreement with the usual expectation for fire retardancy. The time to PHRR is longer for PMMA-LDH nanocomposites, compared to pristine PMMA, while it is constant for the copolymer containing LDH.

In the presence of MMT, there is very little difference between PMMA and its copolymer. The reductions in the PHRR are slightly larger for the copolymer but only slightly larger. Since the PHRR is relatively constant, one might expect that the mass loss rate would also be similar and this is observed. The usual explanation for the reduction in the PHRR is that the decrease in the mass loss rate is the cause of the reduction in the PHRR.

Normally only $3 \% \mathrm{MMT}$ is added to a polymer because this amount has proved optimal for most purposes. In previous work, we have used between 0.1 and 5\% MMT with polystyrene and found that there are only marginal differences over this concentration range ${ }^{[30]}$. It is very surprising that for PMMA the PHRR continues to decrease as MMT is added. This suggests that the entire body of work on the various polymers with MMT must be reexamined to determine if there is a concentration effect and to determine the origin of this effect.

Barrier formation during combustion is the general mechanism invoked for fire retardancy using polymer-clay nanocomposites. Char formation and the char structure are very important for fire retardant efficiency; photographs of the char after cone calorimetry are shown in Fig. 9. The char mass and structure are very different between polymer and copolymer nanocomposites. There is no char formation for either pristine, commercial PMMA or suspension polymerized PMMA, but the suspension copolymerized material containing no additive retains carbonaceous char, with a mass of $2 \%$ and a network structure (Fig. 9C). For the LDH-containing PMMA system, the char content of the composite with $5 \%$ HDEHP-LDH loading is $2.1 \%$ and only a part of the aluminum foil in which the cone sample was wrapped can be seen at the end of the run (Fig. 9A). The copolymer with $5 \% \mathrm{LDH}$ gives $8.2 \%$ char and this covers the entire aluminum foil (Fig. 9D). Even at 3\% LDH loading, the entire aluminum foil is covered with residue. 

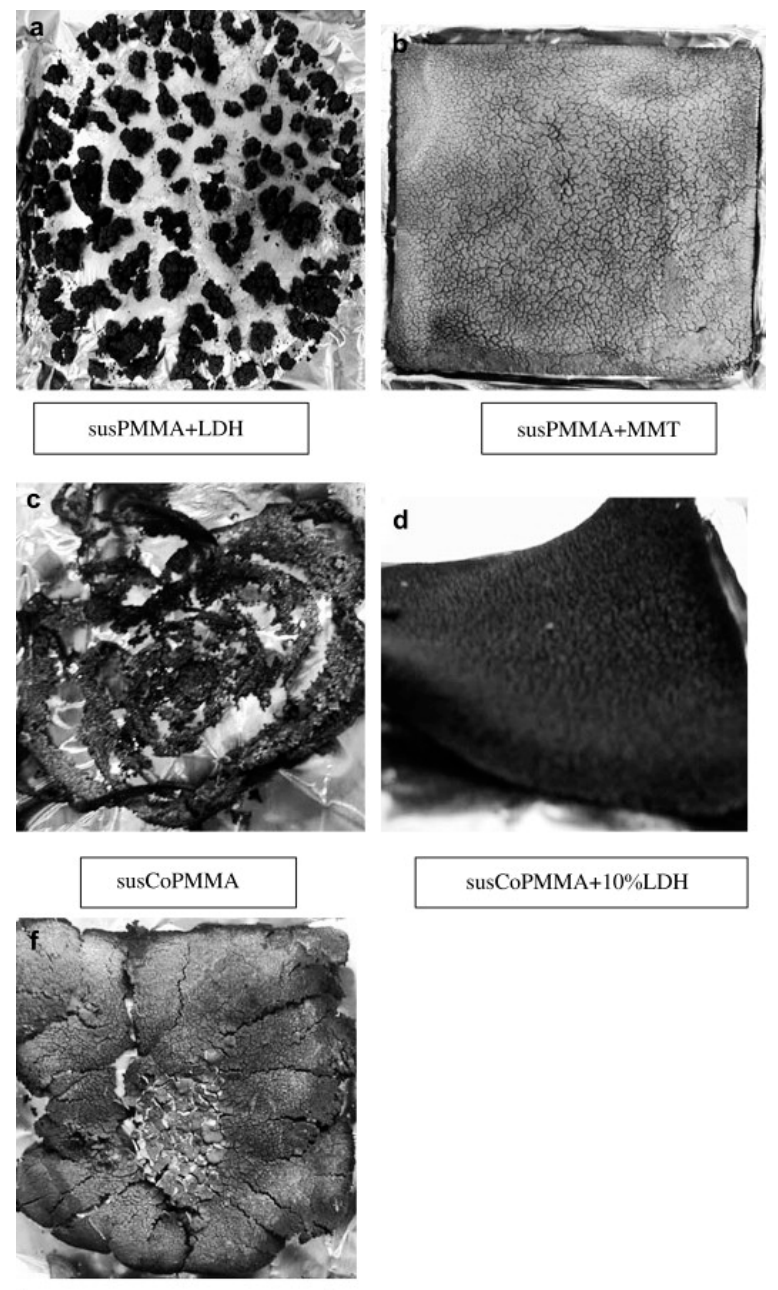

susCoPMMA+10\%LDH

sus CoPMMA+MMT

Fig. 9. Photographs of residual char from (a) susPMMA + LDH, (b) susPMMA + MMT, (c) susCoPMMA, (d) susCoPMMA + 10\% LDH and (e) sus CoPMMA + MMT formulations.

The copolymer nanocomposites form a unitary char structure even at very low LDH loading (3\%) and this char covers all of the aluminum foil. For the $5 \%$ MMT system, the char content for the PMMA nanocomposite is $7.5 \%$, and the char content increases to $11.5 \%$ for the copolymer, and both have a seemingly impermeable network structure. The significantly higher char content for the copolymer nanocomposites, compared to PMMA, may imply some synergy to enhance cross-linking and the formation of carbonaceous char.

The XRD traces of the residual char after cone calorimetry shows only very weak peaks before calcination; after calcination at $900{ }^{\circ} \mathrm{C}$ for $12 \mathrm{~h}$, the XRD peaks sharpen and the pattern is shown in Fig. 10. For SusCoPMMA with no additive, complete volatilizationoccurred after calcination. The main phase in the formulation sus PMMA + LDH char is magnesiumphosphate $\left(\mathrm{Mg}_{3}\left(\mathrm{PO}_{4}\right)_{2}\right)$ with a small amount of spinel $\left(\mathrm{Mg}_{0.4} \mathrm{Al}_{0.6}\right) \mathrm{Al}_{1.8} \mathrm{O}_{4}$. The char of copolymer nanocomposites with either $\mathrm{LDH}$ or MMT consists mainly of aluminum phosphate $\left(\mathrm{AlPO}_{4}\right)$. These data agree with analysis which shows the presence of phosphorus in the residue. 


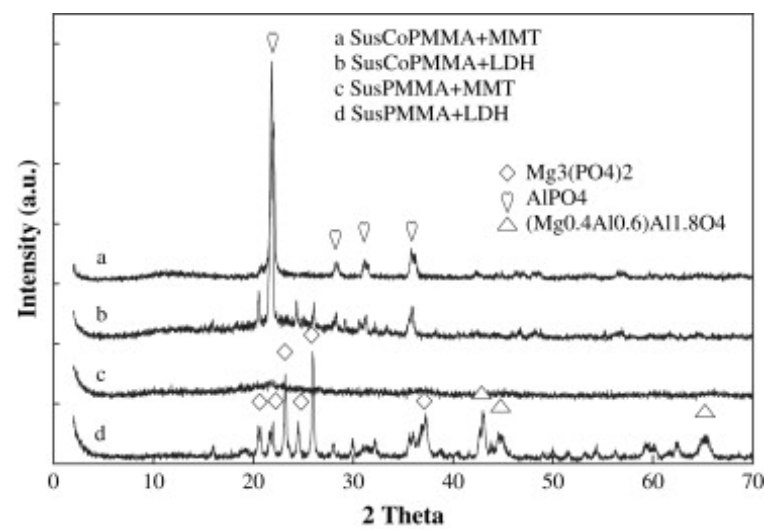

Fig. 10. XRD traces of the residual char calcined at $900{ }^{\circ} \mathrm{C}$ for $24 \mathrm{~h}$.

\section{Conclusions}

Both the PMMA homopolymer and its copolymer with bis[2-(methacryloyloxy)ethyl] phosphate were synthesized and combined with two nano-dimensional materials, an LDH and an MMT. The presence of the phosphorus-containing comonomer enables the dispersion of the additives in the polymer. MMT is much better dispersed in both the polymer and the copolymer than is the LDH. For the systems PMMAMMT, the reduction in the peak heat release rate continues to increase above $3 \%$ loading, which is behavior unlike that of other polymers that have been studied and suggests that there are effects in these systems that are still not understood.

\section{References}

[1] Hu Y, Song L. Nanocomposites with halogen and nonintumescent phosphorus flame retardant additives. In: Morgan AB, Wilkie CA, editors. Flame retardant polymer nanocomposites. Hoboken, New Jersey: John Wiley and Sons, Inc.; 2007. p. 191-234.

[2] Green J. A review of phosphorus-containing flame retardants. J Fire Sci 1996;14:353-66.

[3] Wang CS, Shieh JY, Sun YM. Phosphorus containing PET and PEN by direct esterification. Eur Polym J 1999;35:1465-72.

[4] Ebdon JR, Price D, Hunt BJ, Joseph P, Gao F, Milnes GJ, et al. Flame retardance in some polystyrenes and poly(methyl methacrylate)s with covalently bound phosphorus-containing groups: initial screening experiments and some laser pyrolysis mechanistic studies. Polym Degrad Stab 2000;69:267-77.

[5] Delaviz Y, Gungor A, McGrath JE, Gibson HW. Phosphine oxide-containing aromatic polyester. Polymer 1992;33:5346-7.

[6] Lee FT, Green J, Gibilisco RD. Recent developments using phosphoruscontaining diol as a reactive combustion modifier for rigid polyurethane foams - part III. J Fire Sci 1984;2:439-53.

[7] Sivriev C, Zabski L. Flame retarded rigid polyurethane foams by chemical modification with phosphorus- and nitrogen-containing polyol. Eur Polym J 1994;30:509-14.

[8] Buckingham MR, Lindsay AJ, Stevenson DE, Muller G, Morel E, Costes B, et al. Synthesis and formulation of novel phosphorylated flame retardant curatives for thermoset resins. Polym Degrad Stab 1996;54:311-5. 
[9] Levchik SV, Camino G, Costa L, Luda MP. Mechanistic study of thermal behavior and combustion performance of carbon fiber-epoxy resin composites fire retarded with a phosphorus-based curing system. Polym Degrad Stab 1996;54:317-22.

[10] Price D, Pyrah K, Hull TR, Milnes GJ, Ebdon JR, unt BJ, et al. Flame retarding poly(methyl methacrylate) with phosphorus-containing compounds: comparison of an additive with a reactive approach. Polym Degrad Stab 2001;74:441-7.

[11] Price D, Bullett KJ, Cunliffe LK, Hull TR, Milness GJ, Ebdon JR, et al. Cone calorimetry studies of polymer systems flame retarded by chemically bonded phosphorus. Polym Degrad Stab 2005;88:74-9.

[12] Morgan AB, Wilkie CA, editors. Flame retardant polymer nanocomposites. Wiley-Interscience; 2007.

[13] Gilman JW, Jackson CL, Morgan AB, Harris Jr R, Manias E, Giannelis EP, et al. Flammability properties of polymer-layered-silicate nanocomposites. Polypropylene and polystyrene nanocomposites. Chem Mater 2000;12:1866-73; Chen K, Wilkie CA, Vyazovkin S. Revealing nano-confinement in degradation and relaxation studies of two structurally different polystyrene-clay systems. J Phys Chem B 2007;111:12685-92.

[14] Costache MC, Heidecker MJ, Manias E, Camino G, Frache A, Beyer G, et al. The influence of carbon nanotubes, organically modified montmorillonites and layered double hydroxides on the thermal degradation and fire retardancy of polyethylene, ethylene-vinyl acetate copolymer and polystyrene. Polymer 2007;48:6532-45.

[15] Wang DY, Zhu J, Yao Q, Wilkie CA. A comparison of various methods for the preparation of polystyrene and poly(methyl methacrylate) clay nanocomposites. Chem Mater 2002;14:383743.

[16] Zhu J, Wilkie CA. Thermal and fire studies on polystyrene-clay nanocomposites. Polym Int 2000;49:1158-63.

[17] Jash P, Wilkie CA. Effects of surfactants on the thermal and fire properties of poly(methyl methacrylate)/clay nanocomposites. Polym Degrad Stab 2005;88: 401-6.

[18] Erickson KL, Bostrom TE, Frost RL. A study of structural memory effects in synthetic hydrotalcites using environmental SEM. Mater Letters 2005;59: 226-9.

[19] Zammarano M, Franceschi M, Bellayer S, Gilman JW, Meriani S. Preparation and flame resistance properties of revolutionary self-extinguishing epoxy nanocomposites based on layered double hydroxides. Polymer 2005;46: 9314-28.

[20] Manzi-Nshuti C, Wang D, Hossenlopp JM, Wilkie CA. Aluminum-containing layered double hydroxides: the thermal, mechanical and fire properties of (nano)composites of poly(methyl methacrylate). J Mater Chem 2008;18: 3091-102.

[21] Nyambo C, Songtipya P, Manias E, Jimenez-Gasco M, Wilkie CA. Effect of MgAllayered double hydroxide exchanged with linear alkyl carboxylates on fire retardancy of PMMA and PS. J Mater Chem 2008;18:4827-38.

[22] Manzi-Nshuti C, Wang D, Hossenlopp JM, Wilkie CA. The role of the trivalent metal in an LDH: synthesis, characterization and fire properties of the thermally stable PMMA/LDH system, submitted.

[23] Wang L, Chen D, Su S, Wilkie CA. Variation of anions in layered double hydroxides: effects on dispersion and fire properties, submitted. 
[24] Nyambo C, Kandare E, Wang D, Wilkie CA. Flame-retarded polysthrene: investigating chemical interactions between ammonium polyphosphate and MgAl layered double hydroxide. Polym Degrad Stab 2008;93:1656-63.

[25] Zhao HT, Kathryn LN. Dodecyl sulfate-hydrotalcite nanocomposites for trapping chlorinatedorganic pollutants in water. J Colloid Interface Sci 2004;274: 613-24.

[26] Marezenko Z. Spectrophotometric determination of elements. Chichester: Haslet Press; 1976. p. 425.

[27] Holland BJ, Hay JN. The effect of polymerization conditions on the kinetics and mechanisms of thermal degradation of PMMA. Polym Degrad Stab 2002;77: 435-9.

[28] Kashiwagi T, Inaba A, Brown JE, Hatada K, Kitayama T, Masuda E. Effect of weak linkages on the thermal and oxidative degradation of poly(methyl methacrylates). Macromolecules 1986;19:2160-86.

[29] Green J. Phosphorus containing flame retardants. In: Grand AF, Wilkie CA, editors. Fire retardancy of polymeric materials. New York: Marcel Dekker; 2000. p. 147-70.

[30] Zhu J, Uhl FM, Morgan AB, Wilkie CA. Studies on the mechanism by which the formation of nanocomposites enhances thermal stability. Chem Mater 2001; 13:4649-54. 